\title{
Potential Suport Vector Machines and Self-Organizing Maps for phytoplankton discrimination
}

\author{
Ismael F. Aymerich, Jaume Piera and Aureli Soria-Frisch
}

\begin{abstract}
Fluorescence spectroscopy is a powerful technique usually used to evaluate phytoplankton marine environments. In this study, a kernel method (Potential Support Vector Machine, P-SVM) is presented, evaluating its capability to achieve phytoplankton classification from its fluorescence spectra. Different phytoplankton species were studied, and their fluorescence spectra were acquired in laboratory. In a previous study working with Self-Organizing Maps (SOM), it was proved with experimental data from laboratory that excitation spectra were more discriminative than emission spectra. It was also shown that using some preprocessing techniques, such as derivative analysis, the classification performance from emission fluorescence data can be improved. The classification results were encouraging to keep working with emission fluorescence, and herein we present a comparison between P-SVM and SOM for this goal.
\end{abstract}

\section{INTRODUCTION}

$\mathrm{T}$ HE use of hyperspectral sensors in marine studies has increased considerably in the last years. Fluorescence spectroscopy, for example, has rapidly become a powerful tool for characterizing phytoplankton communities in marine environments. Using different fluorescence spectra techniques, it is now possible to discriminate the major phytoplankton groups. It is a noninvasive technique able to measure water properties directly, and provide qualitative and quantitative information about phytoplankton. Hyperspectral sensors provide the potential for more accurate and detailed information extraction than possible with any other type of sensed data.

Nowadays, several studies use phytoplankton fluorescence spectroscopy to discriminate between different phytoplankton algal groups [1][2]. Several studies have been carried out since Yentsch and Pinney [3] proposed an ataxonomic technique that utilized the spectral fluorescence signatures of major ocean phytoplankton to study their population structure in 1985. In some of these works high taxonomic discrimination can be achieved, but they are based on excitation fluorescence measurements, which

Manuscript received February 7, 2010. This work was supported in part by the project ANERIS (PIF-015-1), funded by the Spanish National Research Council (CSIC).

I. F. Aymerich is with the Marine Technology Unit (UTM-CSIC), Barcelona, 08003 Spain (corresponding author phone: +34 932309500; email: ismaelf@utm.csic.es.

J. Piera, is with the Marine Technology Unit (UTM-CSIC), Barcelona, 08003 Spain (e-mail: jpiera@utm.csic.es).

Soria-Frisch is with Starlab, Barcelona, 08035, Spain (e-mail: aureli.soria-frisch@starlab.es). require stimulation at different wavelengths and thus considerable time to obtain the complete spectral profile. This time requirement means a limitation in the number of samples acquired and, in consequence, a low vertical and horizontal resolution. Although low resolution is not a handicap for some studies, there are cases where the time acquisition is an important constraint [4] and these types of techniques may not be valid and it is necessary a faster acquisition method. The use of hyperspectral sensors lets acquire fluorescence spectra almost instantly. Working with emission fluorescence spectra instead of excitation fluorescence spectra, the question to be addressed is if the information contained in emission fluorescence spectra is high enough to discriminate among different species, or at least phytoplankton groups.

Many supervised methods have been developed to handle the multi- and hyperspectral data classification problem. Artificial neural networks have been successfully used for multispectral data classification. In the recent years, a kernel method, Support Vector Machines (SVMs) [5] has been successfully used for hyperspectral data classification [6]. The properties of SVMs make them well-suited to tackle the problem of hyperspectral classification since it can: a) handle large input spaces efficiently; b) deal with noisy samples in a robust way; and c) produce sparse solutions. Kernel methods can be applied for linear and nonlinear classification.

In this paper, we present a comparison between the classification results from hyperspectral data based on Potential-Support Vector Machines (P-SVM) [7], a kernel method based on SVMs, and those obtained using a neural network method, SOM, that was previously presented in [8].

\section{MATERIAL AND METHODS}

In [8] a classification method for phytoplankton fluorescence data using SOM was presented. The following sections briefly describe the data used for this study, introduce the P-SVM method and present the results obtained with P-SVM compared with those obtained using SOM.

\section{A. Potential Support Vector Machines (P-SVM)}

P-SVM is a supervised learning method used for classification and regression. As well as standard Support Vector Machines, it is based on kernels. The P-SVM is a large margin method that uses an objective function, which 
minimizes a scale-invariant capacity measure, and several constraints, which enforce a low empirical error. In contrast to standard support vector machines approaches, the P-SVM can also handle negative definite and non-square kernel matrices. The main advantage of P-SVM in our application is the fact that P-SVM needs less "support features" than standard SVM for evaluating new data samples [7].

As well as SVM, P-SVM performs binary classifications, and its implementation addressing multi-class classification problems can be approached in two ways. The first one consists of defining an architecture made up of an ensemble of binary classifiers. The decision is then taken by combining the partial decisions of the single members of the ensemble. The second one is represented by SVMs formulated directly as a multi-class optimization problem. The number of parameters to be estimated considerably increases in a multi-class optimization formulation, because of the number of classes that are to be simultaneously discriminated. This renders the method less stable and, accordingly, affects the classification performances in terms of accuracy. For this reason, multi-class optimization is not as successful as the approach based on the two-class optimization [9].

\section{B. Self-Organizing Maps classification}

Self-Organizing Maps (SOM) [10] are a type of unsupervised ANNs (Artificial Neural Network). It is particularly suitable for visualization of high-dimensional feature spaces because the SOM method projects highdimensional data on a low dimensional grid, usually two dimensional. This projection can thence be used for classification.

Although Self-Organizing Maps is an unsupervised classification methodology, it is possible to use it for labelling unknown data as any supervised classification methodology

TABLE I

PHYTOPLANKTON CULTURES UNDER STUDY

\begin{tabular}{llcc}
\hline \multicolumn{1}{c}{ Species } & Division & Abbreviation & $\begin{array}{c}N^{\boldsymbol{o}} \\
\text { Samples }\end{array}$ \\
\hline $\begin{array}{l}\text { Alexandrium } \\
\text { minutum }\end{array}$ & Dinophyceae & Am & 41 \\
\hline $\begin{array}{l}\text { Thalassiosira } \\
\text { weissflogii }\end{array}$ & Bacillariophyceae & Thwi & 39 \\
\hline $\begin{array}{l}\text { Dunaliella } \\
\text { primolecta }\end{array}$ & Chlorophyceae & Duna & 40 \\
\hline $\begin{array}{l}\text { Isochrysis } \\
\text { galbana }\end{array}$ & Prymnesiophyceae & Iso & 30 \\
\hline $\begin{array}{l}\text { Pleurochrysis } \\
\text { elongata }\end{array}$ & Prymnesiophyceae & P1 & 42 \\
\hline
\end{tabular}

as shown in [8]. Hence one can train it in an unsupervised manner and then label the trained output map with a ground truth data set. Thence the resulting labelled map can be used to classify recall data by finding out the Best Match Unit (BMU). The label of a recalled sample will correspond to this of the corresponding BMU.

Once we can classify in a supervised manner based on the SOM output map as formerly described, the generation of any performance measure, e.g. confusion matrix, TPR/FPR, Kappa, is straight forward.

\section{Fluorescence Data}

Five different strains from different taxonomic groups of phytoplankton were used in the present work (Table I). The cultures were incubated at $20^{\circ} \mathrm{C}$ in a $\mathrm{f} / 2$ medium under a $12 \mathrm{~h}$ dark - $12 \mathrm{~h}$ light cycle. The Aminco-Bowman Series 2 Spectrometer was used to perform the measurements of fluorescence in the laboratory. The cultures were sampled in a $1 \mathrm{~cm}$ quartz cuvette. The data acquisition was done during two separated periods of time, so two data sets from each specie were obtained. The samples were taken every day with a slit width of $4 \mathrm{~nm}$ and a scan wavelength speed of 20 $\mathrm{nm} / \mathrm{s}$. Due to the different growth speed between the cultures not all groups have the same number of samples.

For this study the first two days were not taken into account due to its low culture concentration and fluorescence signal. The ranges of excitation and emission wavelength were 200-600 nm every $10 \mathrm{~nm}$ and 200-800 nm every $1 \mathrm{~nm}$, respectively. The Matrices measured have 41 rows and 601 columns. An example of one of these matrices is shown in Fig. 1, where the diagonal peaks appreciated correspond to the effects of Raman and Rayleigh scatters.

\section{Selecting and preparing data}

As it has been mentioned, it has been acquired a replica from each specie and the total number of samples is shown in Table I. From the acquired data, two different sets within this work can be distinguished: the first one taking into account

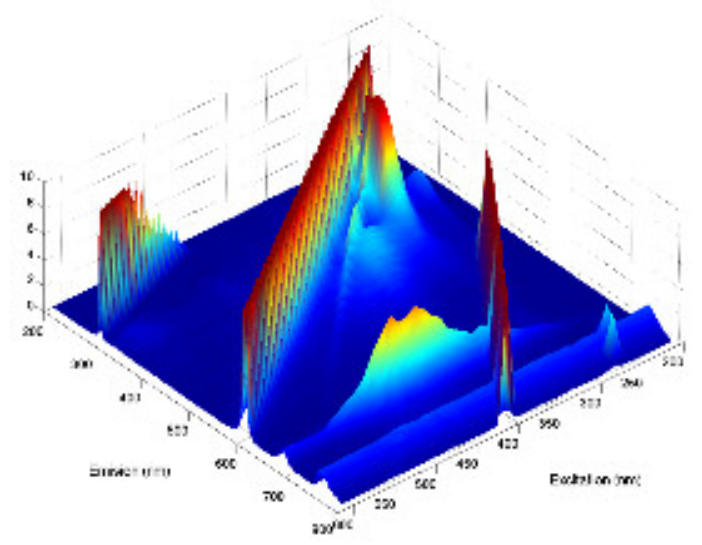

Fig. 1. Example of an Excitation-Emission Matrix (EEM) 
excitation spectra, and the second one using emission fluorescence spectra. The results of both approximations are addressed in this study.

\section{I) Excitation Spectra}

Data consisting of several excitation spectra, where each culture was excited at different wavelengths (the range of selected excitations was between 300 and $600 \mathrm{~nm}$ every 10 $\mathrm{nm}$ ), and its selected emission fluorescence was at $680 \mathrm{~nm}$ for each excitation. This procedure was repeated over several days and replicated again using the same strains. The resulting data set consists of 192 samples of 31 features and in order to choose training and test data leave one out crossvalidation was carried out.

The effect of the Rayleigh scattering peak was studied. Although some studies [2] set it to zero, other approaches such as leaving the peak were evaluated, but, from the results, an interpolation between the two neighbouring samples was the best approximation to avoid this effect obtaining the best results.

\section{II) Emission Spectra}

Using emission spectra, in-Situ acquisition would be faster, and higher vertical and horizontal resolution could be achieved. The main problem is that with excitation spectra you excite and stimulate the different fluorescence pigments at different wavelengths maximizing the information acquired by the sensors, while the emission fluorescence spectra is faster to acquire but you only stimulate the pigments at one wavelength.. It means that differences between fluorescence responses of different phytoplankton classes are expected to be smaller, and therefore the discrimination will be more difficult.

A preliminary study was carried out in order to choose the best excitation wavelength, containing as much information as possible to achieve the best classification. An exploratory classification for each excitation wavelength was made and shown in [8]. The results showed that $490 \mathrm{~nm}$ was the most discriminating wavelength, and from then on it was the excitation wavelength chosen to perform also this study.

Emission fluorescence spectra excited at $490 \mathrm{~nm}$ and ranged 520 and $735 \mathrm{~nm}$ are the data contained on this data set. The range was chosen so as to reduce the dimensionality of the data, and because there is no fluorescence emission at wavelengths below the excitation. The resulting data set consists then of 192 samples of 216 features and the training and test data sets were also chosen using leave one out crossvalidation.

\section{RESULTS AND DISCUSSION}

Discrimination results among five different phytoplankton cultures are now presented. The P-SVM uses C-Support Vector classification with a radial basis function kernel. The optimal cost parameters, $C$, and gamma parameters, g, are determined by performing 8-fold crossvalidation. Since P-SVM is a binary classification method, the multiclass approach to the problem has been implemented using one-against-one approximation, following the max-wins voting strategy (MWV).

A confusion matrix summarizes in a table how well the classifier is working. It shows the number of samples correctly classified and how many have been mistakenly classified. In order to evaluate the performance of both techniques, the Kappa index was computed, extracting as much information as possible from these confusion matrices. This index is a measure of confidence that considers all the elements in the confusion matrix, the diagonal elements as well as the errors of commission (classifying a sample into one class while it belongs to another one) and the errors of omission (classifying a sample belonging to one class into another class). Kappa index can be calculated following (1):

$$
K=\frac{n \sum_{k=1}^{r} X_{k k}-\sum_{k=1}^{r} X_{k+} X_{+k}}{n^{2}-\sum_{k=1}^{r} X_{k+} X_{+k}}
$$

where $\mathrm{n}$ is the total number of samples and $\mathrm{X}_{\mathrm{kk}}$ is the correctly classified samples in class $\mathrm{k}$. If the confusion matrix is considered line by line for class $\mathrm{k}$, then $\mathrm{X}_{\mathrm{k}+}$ is the user's accuracy, whereas a column-by-column analysis specifies the producer's accuracy as $X_{+k}$. The values of Kappa are comprised between 0 and 1, with 1 denoting a perfect classification without any mistake, and 0 the result of a random classification.

TABLE II.

CLASSIFICATION RESULTS FROM EXCITATION FLUORESCENCE SPECTRA: (A) P-SVM, (B) SOM

\begin{tabular}{lrrrrr}
\hline \multicolumn{1}{c}{ (A) } & Thwi & Duna & Pl & Am & Iso \\
\hline Thwi & $\mathbf{1 2}$ & 0 & 22 & 0 & 4 \\
Duna & 1 & $\mathbf{2 7}$ & 11 & 0 & 0 \\
Pl & 0 & 4 & $\mathbf{2 3}$ & 12 & 2 \\
Am & 0 & 0 & 7 & $\mathbf{3 3}$ & 0 \\
Iso & 4 & 13 & 5 & 0 & $\mathbf{7}$ \\
\hline \multicolumn{1}{c}{ (B) } & Thwi & Duna & Pl & Am & Iso \\
\hline Thwi & $\mathbf{3 7}$ & 0 & 0 & 0 & 1 \\
Duna & 2 & $\mathbf{3 6}$ & 0 & 0 & 1 \\
Pl & 19 & 0 & $\mathbf{9}$ & 4 & 9 \\
Am & 7 & 0 & 6 & $\mathbf{2 7}$ & 0 \\
Iso & 2 & 0 & 0 & 0 & $\mathbf{2 7}$ \\
\hline
\end{tabular}




\section{A. Discrimination from Excitation Spectra}

The first part of the experiment was carried out using excitation fluorescence spectra acquired at $680 \mathrm{~nm}$, ranged $300-600 \mathrm{~nm}$ with resolution $10 \mathrm{~nm}$. As it has been previously mentioned, the total number of samples/specie is shown in Table I, containing 31 features each sample. Training and test data sets were chosen using leave one out crossvalidation, and the confusion matrix was computed from the results. Table II shows the resulted confusion matrix.

SOM performs better than P-SVM using excitation spectra. The Kappa indices from both techniques are 0.66015 and 0.42497 , respectively. P-SVM found difficulties classifying Thwi and Iso. It is also remarkable the results obtained with P-SVM in the case Pl and Am, where the classification is significantly better than using SOM.

\section{B. Discrimination from Emission Spectra}

The idea of working with emission spectra comes up from the need of a rapid acquisition method. Acquiring excitation spectra takes almost a second, and the speed of a free falling vertical profiler is near $10 \mathrm{~cm} / \mathrm{s}$. In contrast, acquiring emission fluorescence spectra is almost instantaneous and the number of samples could be increased.

The data set this time consists on emission fluorescence spectra exited at $490 \mathrm{~nm}$ and ranged 520-735 nm with $1 \mathrm{~nm}$ resolution (216 features). Following the same procedure, leave one out cross-validation was used and the results were collected in the confusion matrices shown in Table III.

TABLE III

CLASSIFICATION RESULTS FROM EMISSION FLUORESCENCE SPECTRA: (A) P-SVM, (B) SOM

\begin{tabular}{lrrrcc}
\hline \multicolumn{1}{c}{ (A) } & Thwi & Duna & Pl & Am & Iso \\
\hline Thwi & $\mathbf{1 5}$ & 1 & 21 & 1 & 0 \\
Duna & 6 & $\mathbf{2 9}$ & 4 & 1 & 0 \\
Pl & 3 & 7 & $\mathbf{1 8}$ & 13 & 0 \\
Am & 0 & 0 & 3 & $\mathbf{3 8}$ & 0 \\
Iso & 0 & 7 & 2 & 0 & $\mathbf{2 1}$ \\
\hline \multicolumn{1}{c}{ (B) } & Thwi & Duna & Pl & Am & Iso \\
\hline Thwi & $\mathbf{3 6}$ & 0 & 2 & 0 & 0 \\
Duna & 1 & $\mathbf{3 4}$ & 4 & 1 & 0 \\
Pl & 9 & 6 & $\mathbf{1 6}$ & 9 & 1 \\
Am & 2 & 0 & 6 & $\mathbf{3 3}$ & 0 \\
Iso & 1 & 12 & 0 & 0 & $\mathbf{1 7}$ \\
\hline
\end{tabular}

TABLE IV

CLASSIFICATION RESULTS FROM DERIVATIVE EXCITATION FLUORESCENCE SPECTRA: (A) P-SVM, (B) SOM

\begin{tabular}{lrcccc}
\hline \multicolumn{1}{c}{ (A) } & Thwi & Duna & Pl & Am & Iso \\
\hline Thwi & $\mathbf{2 5}$ & 0 & 11 & 2 & 0 \\
Duna & 0 & $\mathbf{3 4}$ & 2 & 3 & 0 \\
Pl & 7 & 0 & $\mathbf{2 1}$ & 13 & 0 \\
Am & 1 & 0 & 6 & $\mathbf{3 3}$ & 0 \\
Iso & 0 & 0 & 2 & 0 & $\mathbf{2 7}$ \\
\hline \multicolumn{1}{c}{ (B) } & Thwi & Duna & Pl & Am & Iso \\
\hline Thwi & $\mathbf{3 6}$ & 0 & 0 & 2 & 0 \\
Duna & 0 & $\mathbf{3 7}$ & 2 & 0 & 0 \\
Pl & 13 & 2 & $\mathbf{2 0}$ & 5 & 1 \\
Am & 2 & 0 & 10 & $\mathbf{2 8}$ & 0 \\
Iso & 1 & 1 & 0 & 0 & $\mathbf{2 7}$ \\
\hline
\end{tabular}

The performances of both techniques calculated from these matrices are Kpsvm $=0.54252$ and $\mathrm{Ksom}=0.6426$. It is worth noting that while SOM obtained a similar kappa index using excitation spectra, P-SVM improve its performance using emission fluorescence spectra. It still did not obtain comparable results to SOM, but the classification of Am is considerably better.

\section{Derivative Analysis of Hyperspectral Data}

Derivative techniques enhance minute fluctuations in spectra and separate closely related features. But derivatives are notoriously sensitive to noise. Thus, smoothing or otherwise minimizing random noise is a major issue. In this work, a wavelet denoising method [11] has been applied to both data sets before using derivative analysis. Once obtained the derivative spectra, P-SVM and SOM have been applied in order to evaluate the performance using the derivative preprocessing step to enhance their subtle differences.

The resulted confusion matrix from the classification of the derivatives of excitation spectra is shown in Table IV. The computed index Kappa is Kpsvm $=0.68435$. This result is slightly below the value obtained with SOM, Ksom=0.73869.

Applying also derivative analysis to emission fluorescence spectra, P-SVM obtain Kpsvm=0.68091, while Ksom is 0.65617 (Table V). It can be noticed that comparing with the results without derivative, the performance of all species has increased except Iso. In that case the classification results have notably decreased. However, it is 
also remarkable again the performance of P-SVM for Pl and specially for Am.

TABLE V

CLASSIFICATION RESULTS FROM DERIVATIVE EMISSION FLUORESCENCE SPECTRA: (A) P-SVM, (B) SOM

\begin{tabular}{lccccc}
\hline \multicolumn{1}{c}{$(\mathbf{A})$} & Thwi & Duna & PI & Am & Iso \\
\hline Thwi & $\mathbf{2 8}$ & 1 & 8 & 1 & 0 \\
Duna & 0 & $\mathbf{3 1}$ & 9 & 0 & 0 \\
PI & 0 & 3 & $\mathbf{3 3}$ & 5 & 0 \\
Am & 1 & 0 & 2 & $\mathbf{3 8}$ & 0 \\
Iso & 0 & 17 & 1 & 0 & $\mathbf{1 2}$ \\
\hline \multicolumn{1}{c}{$(\mathbf{B})$} & Thwi & Duna & PI & Am & Iso \\
\hline Thwi & $\mathbf{3 7}$ & 1 & 0 & 0 & 0 \\
Duna & 0 & $\mathbf{3 5}$ & 5 & 0 & 0 \\
PI & 3 & 8 & $\mathbf{2 5}$ & 5 & 0 \\
Am & 13 & 0 & 6 & $\mathbf{2 2}$ & 0 \\
Iso & 1 & 10 & 0 & 0 & $\mathbf{1 9}$ \\
\hline
\end{tabular}

\section{CONCLUSION}

This paper shows the results of classification of phytoplankton fluorescence spectra using Potential Support Vector Machines (P-SVM). The performance of this method is then compared with those results obtained with SelfOrganizing Maps (SOM).

From the results can be seen that the phytoplankton information contained in excitation and emission spectra can differ one from each other.

Regarding the classification using P-SVM, it has been implemented doing repeated binary classifications, following the max-wins voting strategy (MWV). It is worth noting that the performance increases using emission fluorescence spectra, but it is also important to mention that using derivative analysis the results $(\mathrm{Kpsvm}=0.68435)$ are comparable with the best results obtained with both techniques $\quad(\mathrm{Ksom}=0.73869$ from derivative excitation spectra). Despite that both techniques have similar results, it has been shown that P-SVM has a significant improvement for two of the five classes (Pleurochrysis elongata - Pl and Alexandrium minutum - Am). This is an important result, since SOM was not able to discriminate these classes.

From the results obtained in this work we can conclude that although SOM performed better than P-SVM, the kernel method is faster in terms of computation classifying samples. This feature is the key in applications where near real time processing must be used. Another characteristic of SVM is the possibility to efficiently deal with a very large number of features due to the exploitation of kernel functions, which makes it an attractive technique to deal with hyperspectral data.

Further work must be done in order to evaluate more precisely the possible potential of P-SVM. Some possible work could be addressed to test our data set with other multiclass approaches than only the max-wins voting strategy. Projective work could also attain the combination of the SOM and P-SVM results for improving the general performance of the system.

\section{ACKNOWLEDGMENT}

This work was supported by the project ANERIS (PIF015-1), funded by the Spanish National Research Council (CSIC).

\section{REFERENCES}

[1] M. Beutler, K. H. Wiltshire, B. Meyer, C. Moldaenke, C. Lüring, M. Meyerhöfer, U-P. Hansen and H. Dau, "A fluorometric method for the differentiation of algal populations in vivo and in situ," Photosynth. Res., 2002, 72, pp. 39-53.

[2] Q-Q. Zhang, S-H. Lei, X-L. Wang, L. Wang and C-J. Zhu, "Discrimination of phytoplankton classes using characteristic spectra of 3D fluorescence spectra," Spectrochim. Acta, 2006, Part A 63, 2, pp. 361.

[3] C. S. Yentsch and D. A. Phinney, "Spectral fluorescence: an ataxonomic tool for studying the structure of phytoplankton populations," Journal of Plankton Research, 1985, 7, 5, pp 617-632.

[4] T. J. Cowles, R.A. Desiderio and S. Neuer, "In situ characterization of phytoplankton from vertical profiles of fluorescence emission spectra," Marine Biology, 1993, 115, 217-222.

[5] C. Cortes and V. Vapnik, "Support-Vector Networks," Machine Learning, 1995, 20, pp.273-297.

[6] C. Dai, X. Huang and G. Dong, "Support Vector Machine for Classification of Hyperspectral Remote Sensing Imagery," FSKD '07: Proceedings of the Fourth International Conference on Fuzzy Systems and Knowledge Discovery, 2007,pp. 77-80.

[7] S. Hochreiter and K. Obermayer, "Support vector machines for dyadic data," Neural Computation, 2006, 18, 6, pp. 1472-1510.

[8] I. F. Aymerich, J. Piera and A. Soria-Frisch. "A rapid technique for classifying phytoplankton fluorescence spectra based on selforganizing maps," Appl. Spectrosc, 2009, 63, pp. 716-726.

[9] F. Melgani and L. Bruzzone, "Classification of Hyperspectral Remote Sensing Images With Support Vector Machines," IEEE Trans. on Geosc. and Remote Sensing, 2004, 42, pp. 1778-1790.

[10] T. Kohonen, The Self-Organizing Map, Proceedings IEEE, 1990, 78, pp 1464-1480.

[11] Piera, J., Quesada, R., Manuel-lazaro, A., Del Rio, J., Shariat Panahi, S. and Olivar, G., "Wavelet denoising technique for high resolution CTD data. Characterization of turbulent oceanic flow," Sensors. Proceedings of the IEEE. 2004, 3, pp1468-1471. 